\title{
Heat and mass transfer in laminated glued timber bar
}

\author{
Aleksandr Ibragimov ${ }^{1,}{ }^{*}$, Lubov Gnedina ${ }^{1}$, Ksenia Zaytseva ${ }^{2}$, Nikita Ushakov ${ }^{1}$, and \\ Konstantin Popkov ${ }^{1}$ \\ ${ }^{1}$ Moscow State University of Civil Engineering (National Research University), 26, Yaroslavskoe \\ shosse, Moscow, 129337, Russian Federation \\ ${ }^{2}$ Military Academy of Nuclear, Biological and Chemical Defense named after Marshal of the Soviet \\ Union of S. K. Tymoshenko, 16, Gorky street, Kostroma, 156015, Russian Federation
}

\begin{abstract}
The main prerequisites for the development of wooden housing construction in Russia are given, which determined the goal of this study to develop a mathematical model of heat and mass transfer (moisture transfer) in glued timber for rational design of enclosing structures. Lamellas can be classified as a capillary - porous body. leaning on theory of academician A.V. Lykov, the formulation of the problem of heat and mass transfer in a multilayer glued laminated timber is presented a system of equations describing non-stationary heat, mass and pressure transfer under real possible operating conditions of enclosing structures is considered. An analytical method is proposed for calculating both individual lamellas and the entire multilayer glued beam. The proposed technique allows the method of solving the inverse problem to directly calculate the resistance value of the entire bar from the unsteady temperature field. The system of equations describing the process is nonlinear and analytically insoluble. To solve the problem, a combined method for solving boundary value problems of heat transfer was used, which is based on a combination of elements of analytical and numerical solutions. A feature of the problem under consideration is that the middle lamella has heat-conducting inclusions in the form of a bough. Method is that the entire heat transfer process is divided into a number of small time intervals. Within each interval, we assume that the temperature is constant at the interface between layers and a constant heat flux through the contacting surfaces, i.e. perfect thermal contact.
\end{abstract}

\section{Introduction}

Target reference point of the national project «Housing and urban environment» in terms of housing commissioning in 2024 is 120.0 million $\mathrm{m} 2$ per year [1]. According to many researchers, including the authors of this article, one of the main sources of growth in the volume of housing construction in Russia should be low-rise wooden housing construction. There are all the prerequisites for this. Russia has the world's largest timber resource base with a volume of 83.4 billion $\mathrm{m} 3$ of timber, which is about a quarter of the world's forest

\footnotetext{
* Corresponding author: igasu_alex@mail.ru
} 
resources. However, the share of the timber industry complex, the main supplier of materials for wooden housing construction, in the total volume of industrial production in Russia has been steadily decreasing in recent years. Although the technical and economic feasibility of wooden housing construction is obvious, at present in Russia wooden houses account for about $10-15 \%$ of the total volume of housing produced in the country, i.e., about 6.0 million $\mathrm{m} 2$ [2].

Despite the high urbanization in the Russian Federation, according to data [3] up to 20\% of the population lives in wooden houses, i.e. one in five Russians wooden house - "sweet home". The rest of the population is genetically inherent in the desire to own their own environmentally friendly (preferably wooden) home. Until 31.12.2024, the expansion of the use of wooden structures should be ensured, taking into account the best practices in construction in the pilot regions (see clause 1.7 [4]).

Analysis of the current situation allows us to conclude that it is necessary to revive wooden housing construction on a qualitatively new basis, using modern technologies, traditions, accumulated colossal experience.

Wood, although a renewable resource, requires careful handling. The uncontrolled and gross supply of "round timber" abroad must be limited as much as possible and not raw materials and materials should be supplied for export, but products and structures. Wood glued structures can undoubtedly solve this problem.

January 14, 11:45 RIA News. The Accounts Chamber identified problems in the export of raw timber from the country. September 30, 2020 - RIA Novosti. At a meeting on the development and decriminalization of the forestry complex, Russian President Vladimir Putin ordered a complete ban on the export of unprocessed coniferous and valuable hardwood timber from the country. According to him, it comes to the export ban "rough or rude, just kind of treated softwood and hardwood valuable." The ban should be introduced from January 1, 2022. Also, the head of state pointed out the need to revise the approaches to the work of customs, so that "round timber" is not exported under the guise of sawn timber. Putin demanded to make the entire chain from logging to the manufacture of finished products as transparent as possible in order to prevent loopholes for crime. From January 1 of the future (2021), the system of accounting for timber and transactions with it should be launched in a pilot mode, and from July 1 - become mandatory. The President also instructed to launch from 2021 soft loans for small and medium-sized businesses for the re-equipment of production facilities and the creation of facilities for deep wood processing. According to Putin, a special modernization program for small and mediumsized businesses will be launched on the basis of the Industrial Development Fund, it should start on January 1 next year.

Currently, the Russian industry produces a fairly large range of glued materials and wood products. Bonding helps to increase the strength properties of wood materials, improve the appearance, obtain large format products, more economical use of wood raw materials, etc. This led to the widespread use of glued timber dwelling houses [3]. The purpose of this study was to develop a mathematical model of heat and mass transfer (moisture transfer) in glued timber for the rational design of enclosing structures.

\section{Materials and methods}

Glued laminated timber consists of several layers - lamellas. Lamellas can be classified as a capillary - porous body. In capillary-porous bodies, moisture transfer occurs in the direction from the highest potential to the lowest. The moisture flux density is directly proportional to the gradient of the mass transfer potential (hereinafter, letter designations are taken according to [5]. 


$$
\vec{J}_{m}=-\lambda_{m} \nabla \Theta
$$

The mass transfer described by expression (1) is called mass conductivity, it characterizes the molecular transfer of vapor and the transfer of liquid under the action of capillary and osmotic forces.

Interrelated transfer of heat and mass in the solid described by the system of equations in partial derivatives of the form [6]:

$$
\begin{aligned}
& \frac{\partial t}{\partial \tau}=a_{q} \nabla^{2} t+\varepsilon r \frac{c_{m}}{c_{q}} \frac{\partial \Theta}{\partial \tau}+\frac{c_{p} k}{c_{q} \gamma_{0}} \nabla P \nabla t, \\
& \frac{\partial \Theta}{\partial \tau}=a_{m} \nabla^{2} \Theta+a_{m} \delta \nabla^{2} t+a_{m} \delta^{\prime \prime} \nabla^{2} P \\
& \frac{\partial P}{\partial \tau}=a_{p} \nabla^{2} P-\varepsilon \frac{c_{m}}{c_{i n}} \frac{\partial \Theta}{\partial \tau} .
\end{aligned}
$$

The boundary conditions on the body surface are:

$$
\begin{gathered}
-\lambda_{q}(\nabla t)_{n}+q_{q}(\tau)-(1-\varepsilon) r q_{m}(\tau)=0, \\
\lambda_{m}(\nabla \Theta)_{\text {sur }}+\lambda_{m} \delta^{\prime}(\nabla t)_{\text {sur }}+\lambda_{p}(\nabla P)_{s u r}+q_{m}(\tau) \\
P_{c}=p=\mathrm{const}
\end{gathered}
$$

The influence of the barodiffusion effect for building structures is small, therefore, in engineering calculations, it is often neglected, then system (2) - (7) is simplified, since equation (4), condition (7), and the third term in expression (6) drop out of it vanishes.

In the particular case, when the influence of the effects of thermal diffusion and internal phase transformations is negligible and the main mass transfer is carried out by means of mass conductivity, the last terms of the right-hand side disappear in equations (2) and (3). The system of equations of interconnected heat and mass transfer breaks down into two independent problems of heat and mass transfer, which are described by parabolic equations of heat conduction.

First term of expression (5) $-\lambda_{q}(\nabla t)_{\text {sur }}$ represents the amount of heat received from the surface into the body by thermal conductivity; The second term $q_{q}(\tau)$ corresponds to the amount of heat supplied to the body surface; The third term $(1-\varepsilon) r q_{m}(\tau)$ represents the amount of heat used to vaporize the liquid. If evaporation occurs only inside the body $(\varepsilon=1)$, then the third term vanishes; in the physical sense, only vapor is supplied to the surface of the body. When $(\varepsilon=0)$ - only liquid is supplied to the surface of the body (moistening the outer walls with rainwater), then evaporation occurs only on the surface of the body. Expression (6) is the mass balance equation for a substance. The physical meaning is that the flow of moisture mass is diverted from the body surface to the environment $q_{m}(\tau)$, and moisture is supplied to the body surface due to the gradients of the mass transfer potential $\lambda_{m}(\nabla \Theta)_{\Pi}$, heat transfer $\lambda_{m} \delta^{\prime}(\nabla t)_{\mathrm{c}}$ and general pressure $\lambda_{p}(\nabla P)_{\mathrm{c}}$. Expression (7) represents the real-life equality of the pressure of the vapor-gas mixture at the surface of the body and the barometric pressure of the environment.

At a given heat flow $q_{q}(\tau)$ and moisture $q_{m}(\tau)$ boundary conditions (5) and (6) are boundary conditions of the second kind.

If we set the law of interaction of a body with moist air: 


$$
\begin{aligned}
& q_{q}(\tau)=\alpha_{q}\left(t_{c}-t_{n}\right) \text { - Newton's law, } \\
& q_{m}(\tau)=\alpha_{m}\left(\Theta_{n}-\Theta_{c}\right) \text { - Dalton's law, }
\end{aligned}
$$

and substitute expressions (8) and (9) in conditions (5) and (6), then the boundary conditions of the third kind are obtained.

If the heat transfer coefficients $\alpha_{q}$ and mass transfer $\alpha_{m} \rightarrow \infty$ or $\lambda_{p}$ and $\lambda_{m} \rightarrow 0$, then the boundary conditions of the first kind are obtained from the boundary conditions of the third kind.

Boundary conditions of the fourth kind reflect the ideal thermal and mass contact of the contacting surfaces:

$$
\begin{aligned}
& t_{i}=t_{i+1}, \quad \Theta_{i}=\Theta_{i+1} \\
& -\lambda_{q i}(\nabla t)_{c i}=-\lambda_{q(i+1)}(\nabla t)_{c(i+1)}, \quad\left(q_{m i}\right)_{c}=q_{m(i+1) n}
\end{aligned}
$$

Equality (10) represent the equality of heat and mass transfer potentials at the interface $i$ and $\mathrm{i}+1$ layer, and equalities (11) - equality of heat and moisture fluxes.

The heat and mass transfer equations for a one-dimensional system were derived in [7] and have the form:

$$
\begin{aligned}
& \frac{\partial t}{\partial \tau}=a_{q} \nabla^{2} t+\frac{\varepsilon r}{c \gamma} \frac{\partial u}{\partial \tau}, \\
& \frac{\partial u}{\partial \tau}=a_{m} \nabla^{2} u+a_{m} \delta \nabla^{2} t
\end{aligned}
$$

In [7] based on the Kirpichev-Guchman similarity theorem, equations (12) and (13) are written in dimensionless variables and for an unbounded plate in the notation of [7] have the form:

$$
\begin{gathered}
\frac{\partial T(\bar{x}, F o)}{\partial F o}=\frac{\partial^{2} T(\bar{x}, F o)}{\partial x^{2}}-K o * \frac{\partial \Theta(\bar{x}, F o)}{\partial F o} \\
\frac{\partial \Theta(\bar{x}, F o)}{\partial F o}=L u \frac{\partial^{2} \Theta(\bar{x}, F o)}{\partial \bar{x}^{2}}-\operatorname{LuPn} \frac{\partial^{2} T(\bar{x}, F o)}{\partial \bar{x}^{2}}
\end{gathered}
$$

In [7] a method was proposed for solving the system of differential equations (14) and (15) using the Laplace transforms:

$$
F_{L}(\bar{x}, s)=\int_{0}^{\infty} F(\bar{x}, F o) \exp (-s F o) d F o .
$$

The boundary conditions are also reduced to a dimensionless form.

In laminated veneer lumber, the outer panels, as a rule, are protected from moisture on the outer surface. In the inner lamellas, moisture moves within the lamella between the glue lines and does not penetrate through the glue line from one lamella to another. At the same time, an almost perfect thermal contact is created due to the glue seam. Therefore, for laminated veneer lumber, heat engineering, not moisture calculation will be decisive. The presence of moisture in wood can be taken into account by introducing not a constant, but a 
variable value of the coefficient of thermal conductivity $(\lambda)$, taking into account the known regularities $[8,9]$. Then a transition from the system of equations $(12,13)$ to the classical Fourier heat equation is possible.

$$
\frac{\partial t(x, \tau)}{\partial \tau}=a \frac{\partial^{2} t(x, \tau)}{\partial x^{2}} .
$$

Wood is a natural material that includes both knot-free wood and areas with boughs.

In laminated veneer lumber, wood with knots should be placed in the inner lamellas. It has been proved [8] that a knot has a higher thermal conductivity coefficient $(\lambda)$ and, during heat transfer, is a kind of "cold bridge" in relation to knot-free wood. Using the example of a three-layer bar, we will simulate the problem of heat transfer. In the physic-mathematical formulation of the problem of heat transfer through a multilayer fence in a transient mode, we use the technique and designations given in [7].

We have a three-layer bar (see Fig. 1). Heat flux q is applied to the left plane of layer 1. The right plane of layer 3 is adjacent to the outer (sup) environment. The task is to determine the change in temperature $t(x, \tau)$ and heat fluxes $q(x, \tau)$ in time $(\tau)$ and in space along the thickness of the fence $(x)$. The accuracy of the heat engineering calculation for building structures depends on the correctness of the selected values of their thermophysical characteristics. In calculations, two main indicators are usually used coefficients: thermal conductivity $(\lambda)$ and volumetric heat capacity $(\mathrm{c} \rho)$.

For the linear equation of thermal conductivity at $\lambda$ and $c \rho=$ const, the thermal diffusivity coefficient (a) is introduced.

$$
a=\lambda /(c \rho) .
$$

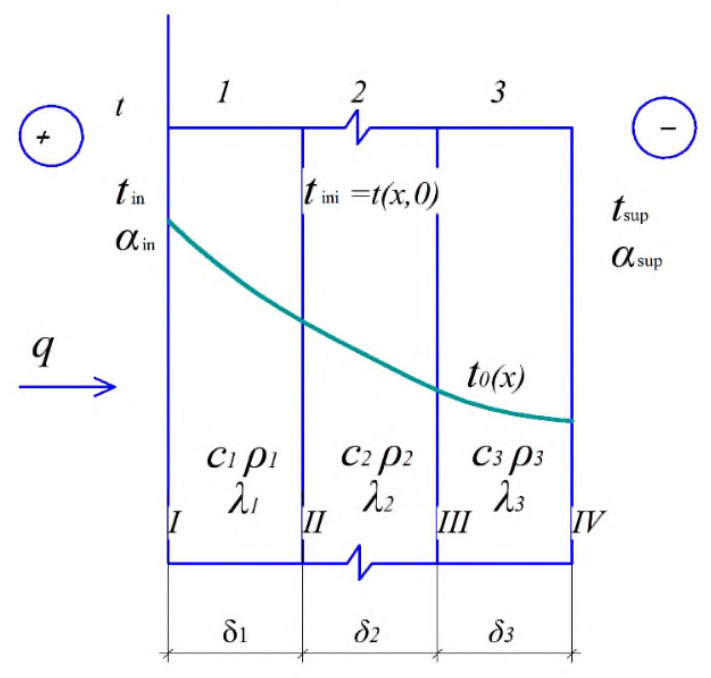

Fig. 1. Temperature field in the bar at the initial moment of time.

To solve the problem, it is necessary that the following be given:

- Initial conditions that determine the temperature distribution over the thickness and at the boundaries of the fence at the initial moment of time;

- Heat conduction equations describing the process of heat transfer through the structure;

- Boundary conditions that determine the conditions for heat transfer on all characteristic planes. 
The initial conditions can be specified in the form of an equation, a table, a graph of the temperature distribution at the time of the start of the process (at $\tau=0$ ). In the general case, the equation of the initial conditions has the form:

$$
\operatorname{tini}=t(x, 0) \text {. }
$$

In general, the heat conduction equation has a nonlinear form:

$$
\frac{\partial[t(x, \tau) c(x, \tau) \rho(x, \tau)]}{\partial \tau}=\frac{\partial}{\partial x}\left[\lambda(x, \tau) \frac{\partial t(x, \tau)}{\partial x}\right]
$$

where the values of the heat capacity coefficient $[\mathrm{c}(\mathrm{x}, \tau) \cdot \rho(\mathrm{x}, \tau)]$ and the thermal conductivity $\lambda(\mathrm{x}, \tau)$ vary from layer to layer, depending on time, temperature and humidity.

For our case, it is convenient to write a system of linear differential equations with constant coefficients. We write each equation for a separate layer with additional boundary conditions at the joints of the layers, assuming that within each separate layer $\lambda \mathrm{i}$ and cipi $=$ const (i - layer number):

$$
c_{i} \rho_{i} \frac{\partial t_{i}}{\partial \tau}=\lambda_{i} \frac{\partial^{2} t_{i}}{\partial x^{2}}
$$

At the boundary II (between 1-st and 2-nd layers), proceeding from the equality of heat fluxes and temperatures, we set conditions of the fourth kind:

$$
\begin{aligned}
& \left.\lambda_{1} \frac{\partial t_{1}}{\partial x}\right|_{I I}=\left.\lambda_{2} \frac{\partial t_{2}}{\partial x}\right|_{I I} \text { or } \mathrm{q}_{1}=\mathrm{q}_{2} \\
& \left.t_{1}\right|_{I I}=\left.t_{2}\right|_{I I} \\
& \text { Boundary conditions on boundary III. }
\end{aligned}
$$

Let us write down the boundary conditions of heat transfer at the boundaries I and IV, i.e. on the inner and outer surfaces of the building envelope, which are in contact with indoor and outdoor air, and are also surrounded by other surfaces. Internal air has a temperature tin, external - tsup. If we take into account convection (convective exchange), then we introduce the coefficient $\alpha \mathrm{k}$, into the calculation, if radiative exchange, then the coefficient $\alpha$ l. If there is a heat source, then the amount of radiant heat absorbed by surface I is determined by the formula:

$$
q c=p \cdot q,
$$

where $\mathrm{p}$ - the absorption coefficient of the fence surface for a given radiation (taken according to the tables of the relevant regulatory documentation, for example, SP 23-1012004. Design of thermal protection of buildings, $q$ is the intensity of the radiation incident on the fence from the source.

If we consider the most general case, then a complex heat exchange occurs on the surface of the fences, which is determined by the conditions of the second kind (a given intensity of the heat flux) and conditions of the third kind (given conditions of heat exchange with the environment). Taking into account the above, the boundary conditions at the boundary I have the form: 


$$
\alpha_{\kappa . i n}\left(t_{\text {in }}-\left.t_{1}\right|_{I}\right)+\alpha_{l . i n}\left(t_{R . i n}-\left.t_{1}\right|_{I}\right)+p_{\text {in }} q_{\text {in }}=-\left.\lambda_{1} \frac{\partial t_{1}}{\partial x}\right|_{I}
$$

here the subscript (in) indicates the inner surface. Boundary conditions at boundary IV have the form:

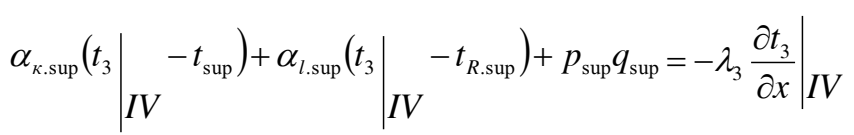

for rooms, the conditions of radiant-convective heat transfer in the practice of construction design are taken into account by a single heat transfer coefficient $\alpha$ in, then condition (24) will take the form:

$$
\alpha_{\text {in }}\left(t_{\text {in }}-\left.t_{1}\right|_{I}\right)=-\left.\lambda_{1} \frac{\partial t_{1}}{\partial x}\right|_{I}
$$

For winter conditions under field studies and laboratory conditions in the absence of heat sources in the laboratory (no heating in summer), a similar simplification can be introduced for the outer surface of the test sample, then condition (25) will take the form:

$$
\begin{aligned}
& \alpha\left(t_{3_{\text {sup }}}\right.
\end{aligned}
$$

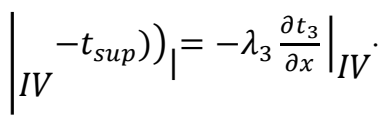

For the summer period, during field studies, it is necessary to take into account solar radiation, during laboratory studies during the heating period - radiation from laboratory heating devices, then instead of (25), a condition of the form should be used:

$$
\alpha_{\text {sup }}\left(\left.t_{3}\right|_{I V}-t_{\text {sup }}\right)+p_{\text {sup }} q_{\text {sup }}=-\left.\lambda_{3} \frac{\partial t_{3}}{\partial x}\right|_{I V}
$$

Condition (28) is a mixed boundary condition of the second and third kind. For convenience, condition (28) can be reduced to boundary conditions of only type III by introducing the conditional temperature of the external environment (tcon):

$$
\alpha_{\text {sup }}\left(t_{\text {con }}-\left.t_{3}\right|_{I V}\right)=-\left.\lambda_{3} \frac{\partial t_{3}}{\partial x}\right|_{I V}
$$

where

$$
\text { tcon }=\text { tsup }+ \text { psup } * \text { sup } / \alpha \text { sup }
$$

So, for our problem, from its general formulation, we choose for further research:

- Initial conditions - expression (19) in the form of a graph of temperature distribution in the thickness of the fence;

- Heat conduction equations of the form (21);

- Border conditions: 
O On the boundary I - condition (24),

○ On the boundary between II and III - condition (22),

○ At the boundary IV - condition (25).

Solving the problem in an analytical way causes great mathematical difficulties, therefore, a mathematical model is proposed that allows them to be avoided and adequately describe the process of unsteady heat transfer in a multilayer structure.

The physical picture of the process can be presented as follows (see Fig.2a): the initial temperature distribution in a multilayer (for simplicity, a three-layer is considered) glued timber corresponds to the expression:

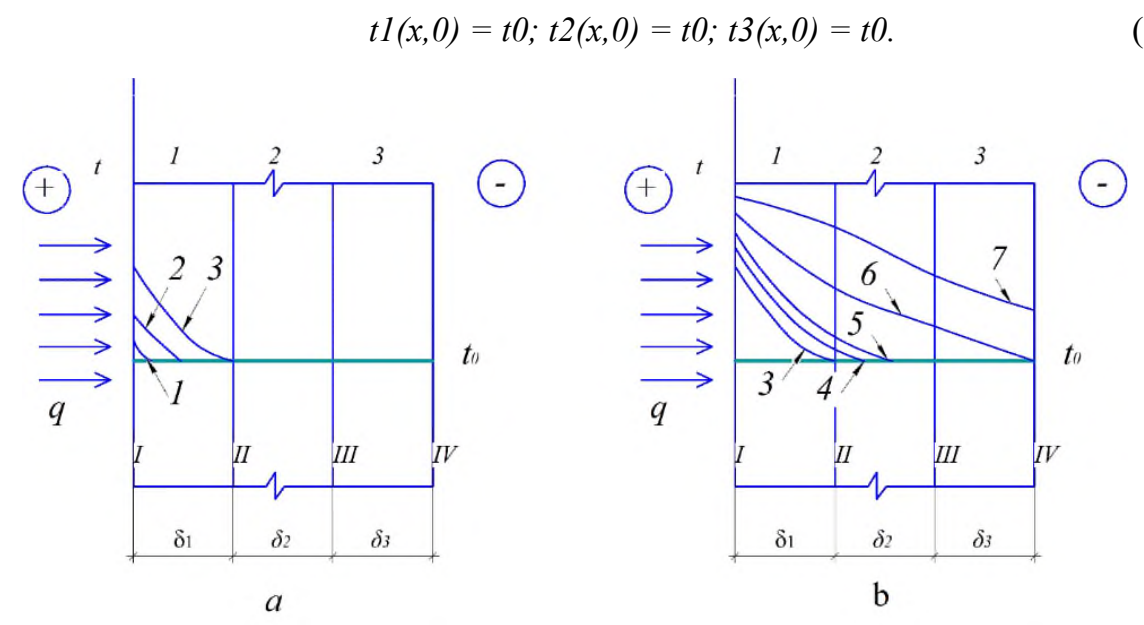

Fig. 2. Distribution of temperature fields in the body of the bar at different times.

At the moment of time $0=0$, a heat flux $q$ is supplied from the left side of the bar, under the influence of which the first layer begins to warm up (see Pic. 2a). The change in the temperature fields are characterized by curves 1 and 2. In this case, the second and third layers will remain with the temperature t0.

At a certain point in time $\tau 1$ (curve 3 ) the heat wave reaches the boundary of the first and second layers, and in this place a temperature gradient appears:

$$
\operatorname{gradt}\left(\delta_{1}, \tau_{1}\right)=-\lambda_{1} \frac{\partial t_{1}\left(\delta_{1}, \tau_{1}\right)}{\partial x}
$$

Note:

Index (1) means the time when the heat wave passes through the entire body of the lamella.

After this moment, the temperature field will penetrate deeper into the timber, as shown by curves 4 and 5 . The temperature of the third layer remains equal to t0 until the moment of time $\tau 2$, when the heat wave reaches the junction of the second and third layers. etc.

At the moment of time $\tau 3$, when the heat wave reaches the outer boundary of the last layer of the enclosure, all layers of the enclosure are involved in the process of heat transfer, and this point in time can be taken as sufficient to determine the resistance to heat transfer $(\mathrm{R})$ in the case of a non-stationary process. With a sufficiently large value of the process time, (theoretically at $\tau \rightarrow \infty$ ) a stationary temperature field will form in the structure (straight broken line 7 in Fig.2b), the values of which are used to calculate R. For the model system under consideration, the following relation will express this: 


$$
R=\frac{1}{\alpha_{1}}+\frac{\delta_{1}}{\lambda_{1}}+\frac{\delta_{2}}{\lambda_{2}}+\frac{\delta_{3}}{\lambda_{3}}+\frac{1}{\alpha_{2}}=\frac{t_{i n}-t_{e n}}{q},
$$

where $\alpha 1, \alpha 2$ - heat transfer coefficient, respectively, of the inner and outer surfaces of the enclosing structure; tin - temperature of the outer surface of the first layer; $t_{e n}$ temperature of the environment from the warm side.

\section{Results}

The proposed technique allows the method of solving the inverse problem to directly calculate the value of $\mathrm{R}$ from the nonstationary temperature field. The system of equations $(19,21,22,24,25)$ is nonlinear and analytically insoluble. To solve the problem, we use a combined method for solving boundary value problems of heat transfer, which is based on a combination of elements of analytical and numerical solutions $[5,6,7,10,11]$. A feature of the presented problem is that the middle lamella has heat-conducting inclusions in the form of a bough (see Fig. 3b).

The essence of the method is that the entire heat transfer process is divided into a number of small time intervals. Within each interval, we assume that the temperature is constant at the boundary of II and III and a constant heat flux through the contacting surfaces, i.e. perfect contact.

The overall task is divided into three autonomous, but interconnected.

Problem 1. Heat transfer in layer 1 with boundary conditions of the third kind, which ensure convective exchange at the boundaries of I, and of the first kind, which characterize the constancy of temperature at the boundaries of II layers 1 and 2.

Problem 2. Heat transfer in layer 2 with boundary conditions of the second kind, which characterize the constancy of the heat flux density through the boundary II, and the first kind, characterizing the constancy of the temperature at the boundary III.

Problem 3. Heat transfer in layer 3 with boundary conditions of the second kind at the boundary III and boundary conditions of the third kind, which characterize the heat transfer between the surface of layer 3 at the boundary IV with the environment according to Newton's law.

Each of these tasks is solved analytically. The solution to the general problem of unsteady heat conduction can be obtained by conjugating these analytical solutions at each time interval. This makes it possible to pass from boundary conditions of the fourth kind to boundary conditions of the first and second kind on the interfaces of layers 1 and 2, 2 and 3, as well as inside layer 2, where a knot is present within the layer, the boundary conditions are both 1 and 2 kind, which makes it easier to solve the problem.

Mathematically, the heat conduction problem for the i-th layer can be written as follows:

$$
\frac{\partial t(x, \tau)}{\partial \tau}=a \frac{\partial^{2} t(x, \tau)}{\partial x^{2}} ; \quad\left(0 \leq x \leq \delta_{i}\right)
$$

where i - layer number.

For problem 1 (Pic.3a), the initial conditions have the form:

$$
t(x, 0)=t_{0}(x)
$$

boundary condition:

$$
t(\delta, \tau)=t_{\delta}
$$




$$
-\lambda_{i} \frac{\partial t(0, \tau)}{\partial x}=\alpha_{\mathrm{in}}\left[t_{c}-t(0, \tau)\right]
$$

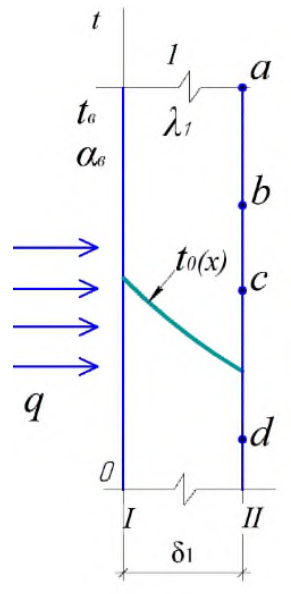

$a$

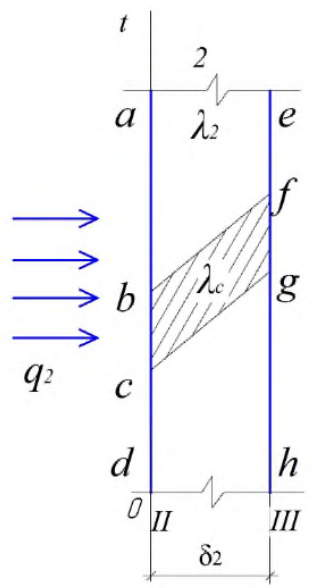

b

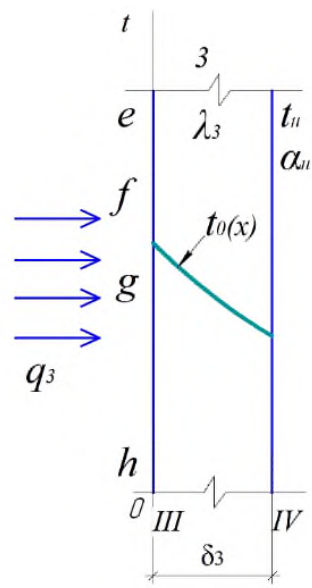

C

Fig. 3. Temperature field in glued timber bar: a - in the first lamella; b - in the second lamella; $c$ - in the third lamella.

Boundary condition (36) shows that surface II of the interface between layers 1 and 2 has a constant temperature t5. Condition (37) characterizes convective heat transfer on the outer surface I.

Note:

Index (1), indicating that the layouts belong to the first layer, is omitted for simplicity.

Problem 2 (Fig. 3b). The condition of problem 2, according to the previously accepted assumptions, can be formulated as follows: heat transfer in an unbounded plate with combined boundary conditions of the second kind on surface II and of the first kind on surface III and no uniform initial conditions.

We place the origin of coordinates at the junction of the layers, i.e. in section II. However, we are considering a middle lamella that has a knot. There are three options for the spatial position of the knot in the lamellae relative to the direction of the heat flow:

- Parallel;

- Orthogonal;

- At an angle.

\section{Discussion}

The mathematical problem of describing heat transfer in the boughs - pure wood system must be considered in a cylindrical coordinate system. Then the solution of boundary value problems is obtained in the form of Bessel functions, the calculation of which is associated with mathematical difficulties. At certain geometric ratios of the lamella thickness to the knot diameter, the heat transfer problem can be replaced by a flat problem. A similar technique was applied in [11].

Within this lamella there are several boundaries: boundary II and boundary III, as well as the boundaries of knot-free wood with a knot along the line bf and cg (fig. 3b). 
Heat transfer inside a lamella with a knot is described in any combination by the boundary conditions 1 and 2 kinds.

Problem 3 (fig. 3c). The condition of Problem 3, according to the previously accepted assumptions, can be formulated as follows: heat transfer in an unbounded plate with combined boundary conditions of the second kind on surface III and of the first kind on surface IV and no uniform initial conditions.

\section{Conclusion}

1. In laminated veneer lumber, moisture moves within the lamella between the glued seams and does not penetrate through the glued seam from one lamella to another. At the same time, an almost perfect thermal contact is created due to the glue seam. Therefore, for laminated veneer lumber, heat engineering, and not moisture calculation will be decisive. The wet state of the material within the lamella can be taken into account in the coefficient of thermal conductivity $(\lambda)$.

2. Resistance to heat transfer of laminated veneer lumber significantly depends on the location of the knots in relation to the direction of the heat flow.

3. The proposed methodology can be used to solve the direct problem, i.e. at the design stage of the structure, taking into account the required resistance to heat transfer, determine the required thickness of the timber or the inverse problem, which is solved during the inspection of buildings - to determine the resistance to heat transfer of the existing structure.

4. Experiments show that even single knots, not to mention whorled ones, significantly reduce the resistance of the structure to heat transfer up to $15 \%$. To calculate glued timber structures, it is necessary to use the reduced heat transfer coefficient, taking into account the knotty parameters. The research carried out by the authors allows this.

5. A feature of the proposed calculation method is that a non-stationary process of heat transfer through a multilayer glued beam is considered, and this makes it possible to simulate the operation of a structure under any initial and boundary conditions at any time interval, adequately assess its resistance to heat transfer and rationally design.

\section{References}

1. Passport of the national project "Housing and Urban Environment" (approved by the Presidium of the Council under the President of the Russian Federation for Strategic Development and National Projects No. 16 dated December 24, 2018).

2. Passport of the federal project "Housing" (approved by the minutes of the meeting of the project committee for the national project "Housing and the urban environment" of December 21, 2018 N 3).

3. Yu.B. Levinskiy, V.I. Onegin, Wooden housing construction (Sankt- Petergburg, 2008)

4. Russian statistical Yearbook (Date of appeal 22.07.2015) access mode: http://www.gks.ru/wps/wcm/connect/rosstat_main/rosstat/ru/statistics

5. V.N. Bogoslovskiy, Construction thermophysics (Heatphysical bases of heating, ventilation and air conditioning), Textbook for universities ("AVOK Severo-Zapad», 2006)

6. V.P. Isachenko, V.A. Isachenko, A.S. Osipova, Heat transfer (M., 1975)

7. A.V. Lykov, Theoretical fundamentals of construction thermophysics (ed. AN BSSR. Minsk, 1961) 
8. A.A. Titunin, V.M. Karavaykov, K.V. Sirotkina, Heat conductivity of wooden glued constructions, Industrial and Civil Engineering magazine 10, 66-67 (2007)

9. B.N. Ugolev, Wood science with fundamentals of forest merchandizing 4-th ed. (M.: MGUL, 2007)

10. S.V. Fedosov, L.Yu. Gnedina, Unsteady heat transfer in multi-layer cladding, inbook problems of building thermal physics of systems for providing microclimate and energy saving in buildings (M.: NIISF, 1999)

11. S.V. Fedosov, V.G. Kotlov, R.M. Aloyan, F.N. Yasinskiy, M.V. Bochkov, Simulation of heat-mass transfer in the gas-solid at dowel joints of timber structures elements. Part 2. Dynamics of temperature fields at arbitrary law of changes of air environment temperature, Construction Materials 8, 73-79 (2014) 